\title{
PROPERTIES OF GALAXIES HOSTING X-RAY-SELECTED ACTIVE GALACTIC NUCLEI IN THE CL1604 SUPERCLUSTER AT $z=0.9$
}

\author{
Dale D. Kocevski ${ }^{1}$, Lori M. Lubin ${ }^{1}$, Brian C. Lemaux ${ }^{1}$, Roy R. GaL ${ }^{1,2}$, Christopher D. Fassnacht $^{1}$, Robin Lin ${ }^{1,3}$, and \\ Gordon K. SQuires ${ }^{1,4}$ \\ ${ }^{1}$ Department of Physics, University of California, Davis, 1 Shields Avenue, Davis, CA 95616, USA; kocevski@ physics.ucdavis.edu \\ 2 Institute for Astronomy, University of Hawaii, 2680 Woodlawn Dr., Honolulu, HI 96822, USA \\ ${ }^{3}$ Siemens Medical Solutions USA, Inc., 1230 Shorebird Way, MS 6-1, Mountain View, CA 94043-1344, USA \\ ${ }^{4}$ Spitzer Science Center, M/S 220-6, California Institute of Technology, 1200 East California Blvd, Pasadena, CA 91125, USA \\ Received 2008 August 27; accepted 2009 May 11; published 2009 July 7
}

\begin{abstract}
Recent galaxy evolution models suggest that feedback from active galactic nuclei (AGNs) may be responsible for suppressing star formation in their host galaxies and the subsequent migration of these systems onto the red sequence. To investigate the role of AGNs in driving the evolution of their hosts, we have carried out a study of the environments and optical properties of galaxies harboring X-ray luminous AGNs in the Cl1604 supercluster at $z \sim 0.9$. Making use of Chandra, HST/ACS and Keck/DEIMOS observations, we examine the integrated colors, morphologies, and spectral properties of nine moderate-luminosity $\left(L_{\mathrm{X}} \sim 10^{43} \mathrm{erg} \mathrm{s}^{-1}\right)$ type 2 Seyferts detected in the Cl1604 complex. We find that the AGNs are predominantly hosted by luminous spheroids and/or bulge-dominated galaxies which have colors that place them in the valley between the blue cloud and red sequence in color-magnitude space, consistent with predictions that AGN hosts should constitute a transition population. Half of the hosts have bluer overall colors as a result of blue resolved cores in otherwise red spheroids and a majority show signs of recent or pending interactions. We also find a substantial number exhibit strong Balmer absorption features indicative of post-starburst galaxies, despite the fact that we detect narrow [O II] emission lines in all of the host spectra. If the [O II] lines are due in part to AGN emission, as we suspect, then this result implies that a significant fraction of these galaxies (44\%) have experienced an enhanced level of star formation within the last $\sim 1 \mathrm{Gyr}$ which was rapidly suppressed. Furthermore we observe that the hosts galaxies tend to avoid the densest regions of the supercluster and are instead located in intermediate density environments, such as the infall region of a massive cluster or in poorer systems undergoing assembly. Overall we find that the properties of the nine host galaxies are generally consistent with a scenario in which recent interactions have triggered both increased levels of nuclear activity and an enhancement of centrally concentrated star formation, followed by a rapid truncation of the latter, possibly as a result of feedback from the AGN itself. Our finding that the hosts of moderate-luminosity AGNs within the Cl1604 supercluster are predominantly a transition population suggests that AGN feedback may play an important role in accelerating galaxy evolution in large-scale structures.
\end{abstract}

Key words: galaxies: clusters: general - large-scale structure of universe - X-rays: galaxies: clusters

Online-only material: color figures

\section{INTRODUCTION}

Several studies have demonstrated that galaxies segregate by rest-frame color, with passively evolving early-type galaxies forming a well defined "red sequence" and gas-rich, starforming systems located in a more diffuse "blue cloud" (e.g., Baldry et al. 2004). This observed color bimodality is commonly thought to be the result of an evolutionary sequence, such that galaxies in the blue cloud quickly migrate onto the red sequence following the termination of star formation, leading to a sparsely populated transition zone between the two regions (Faber et al. 2007; although see Driver et al. 2006 for an alternative view). While simple passive evolution likely contributes to the migration of galaxies from blue to red, recent findings that galaxies exhibit reduced star formation rates as they assemble into regions of even moderate density (Lewis et al. 2002; Gómez et al. 2003) suggest that processes prevalent in richer environments must also play a role in terminating star formation and driving galaxy evolution. Recently merger-driven feedback from active galactic nuclei (AGNs) has been suggested as one such process (Hopkins et al. 2005, 2007; Somerville et al. 2008).
In this scenario, strong gravitational torques produced as a result of galaxy mergers funnel material to the nuclear region of a system, leading to elevated accretion onto the central black hole and enhanced star formation in the form of a nuclear starburst (Barnes \& Hernquist 1991; Mihos \& Hernquist 1996). If sufficiently energetic, the AGN eventually drives outflows that disrupt the host, effectively quenching ongoing star formation by removing the galaxy's gas supply (Springel et al. 2005; Hopkins et al. 2005). As the last generation of newly formed stars fade, the galaxy quickly migrates from the blue cloud to the red sequence, with the activity of the central black hole gradually declining over the same period.

This feedback mechanism provides a method by which the overall properties of a galaxy can be regulated by its central black hole. Indeed there is a growing body of evidence that suggests that AGNs are directly linked to the evolution of their host galaxies. This includes the observed correlation between central black hole mass and the stellar velocity dispersion of a host galaxy's bulge (Gebhardt et al. 2000; Tremaine et al. 2002) and the coeval decline in both the cosmic star formation rate and nuclear activity since $z \sim 1$ (Boyle \& Terlevich 1998). 
If AGNs are directly responsible for driving the evolution of their hosts, then signs of recent or ongoing transformation should be present in these galaxies. This includes disturbed morphologies due to recent interactions, post-starburst signatures resulting from rapidly quenched star formation following a merger-induced enhancement, and colors consistent with the sparsely populated transition zone in the color-magnitude diagram (CMD). While many of these features are observed in the hosts of luminous quasars (QSOs), studies of moderateluminosity Seyferts $\left(L_{\mathrm{X}} \sim 10^{43} \mathrm{erg} \mathrm{s}^{-1}\right)$ in this regard have produced mixed results. For example, while such AGNs are predominantly found in massive, early-type galaxies which have younger stellar populations than their nonactive counterparts (e.g., Kauffmann et al. 2003), only a small fraction shows signs of recent merger activity (De Robertis et al. 1998; Grogin et al. 2005; Pierce et al. 2007). Likewise, although AGNs are found in post-starburst galaxies more often than in normal galaxies (Heckman 2004; Yan et al. 2006; Goto 2006), the fraction of Seyferts associated with post-starburst hosts at low redshift remains fairly small; on the order of $4 \%$ according to a recent study by Goto (2006).

A clearer picture of the prevalence of AGN feedback as an evolutionary driver may be obtained by studying host galaxies in and around large-scale structures at high redshift. In addition to a considerably higher AGN density and galaxy merger rate at $z \sim 1$ relative to the present epoch (Barger et al. 2005; Kartaltepe et al. 2007), there are indications that AGNs are triggered more often in structures than in the field (Gilli et al. 2003; Cappelluti et al. 2005; Eastman et al. 2007; Kocevski et al. 2009), presumably due to the presence of richer, dynamic environments where galaxy interactions are more common (Cavaliere et al. 1992). Several recent wide-field surveys have found that the colors of higher-redshift host galaxies are consistent with a population in transition (Sánchez et al. 2004; Nandra et al. 2007; Georgakakis et al. 2008; Silverman et al. 2008; although see also Westoby et al. 2007) and there are indications that the fraction of AGN hosts exhibiting post-starburst signatures is higher at $z \sim 0.8$ (Georgakakis et al. 2008). Furthermore, Silverman et al. (2008) found that a majority of host galaxies associated with two large-scale structures in the Extended Chandra Deep Field South (E-CDF-S) at $z \sim 0.7$ have colors consistent with the transition zone of the CMD. Coupled with the increased incidence of AGN in such regions, this finding implies that AGN-related feedback may play an important role in accelerating galaxy evolution in large-scale structures.

In this study, we examine the environments and optical properties of galaxies hosting X-ray-selected, moderate-luminosity AGNs in the $\mathrm{Cl1604}$ supercluster at $z=0.9$. The system is the largest structure mapped at redshifts approaching unity, consisting of eight spectroscopically confirmed galaxy clusters and groups and a rich network of filamentary structures. The system spans roughly $10 h_{70}^{-1} \mathrm{Mpc}$ on the sky and $100 h_{70}^{-1} \mathrm{Mpc}$ in depth. The complex structure of the supercluster, as mapped by extensive spectroscopic observations, is described in Gal et al. (2008; hereafter G08) and our X-ray observations of the system are discussed in Kocevski et al. (2009; hereafter K09). The C11604 supercluster is well suited for this study as it provides a diverse set of structures within which AGNs may be preferentially found and a wide range of environments and local conditions that can help constrain the mechanisms responsible for triggering their activity.

In the following sections, we begin by describing our X-ray and optical observations of the Cl1604 system (Section 2) and the process by which we identify AGNs in the supercluster (Section 3). This is followed by an examination of the morphology (Section 4.1), optical colors (Section 4.2), spectral properties (Section 4.3), and environments (Section 4.4) of the galaxies found to host AGNs. We also determine the fraction supercluster members which harbor AGNs and compare this to low-redshift results in Section 5. We discuss our results and their implications for the AGN feedback model in driving galaxy evolution in the Cl1604 system in Section 6. Finally we summarize our results in Section 7. Throughout this paper, all quoted line equivalent widths are in the rest frame, magnitudes are in the AB system, and we assume a $\Lambda$ CDM cosmology with $\Omega_{m}=0.3, \Omega_{\Lambda}=0.7$, and $H_{0}=70 h_{70} \mathrm{~km} \mathrm{~s}^{-1} \mathrm{Mpc}^{-1}$.

\section{OBSERVATIONS AND DATA REDUCTION}

\subsection{X-ray Observations}

Observations of the $\mathrm{Cl} 1604$ supercluster were carried out with Chandra's Advanced CCD Imaging Spectrometer (ACIS; Garmire et al. 2003) on 2006 June 23 (obsID 7343), June 25 (obsID 6933), and October 01 (obsID 6932). A detailed description of the observations and subsequent data reduction are given in $\mathrm{K} 09$, therefore we only provide a brief summary here. The three observations of the supercluster consist of two pointings, one encompassing the northern portion of the system and the other the southern portion, with a 4.9 overlap between the imaged regions. Each pointing was imaged with the $16^{\prime} .9 \times 16^{\prime} .9$ ACIS-I array for total integration times of 19.4, 26.7, and $49.5 \mathrm{ksec}$ for the 7343, 6933, and 6932 data sets, respectively. All three data sets were reprocessed and analyzed using standard CIAO 3.3 software tools and version 3.2 .2 of the Chandra calibration database available through Chandra X-ray Center (CXC). ${ }^{5}$

We searched for point sources using the wavelet-based wavdetect procedure in CIAO, employing the standard $\sqrt{2}^{i}$ series of wavelet pixel scales, with $i=0-16$. Object detection was carried out separately on images in each of the $0.5-2 \mathrm{keV}$ (soft), 2-8 keV (hard), and 0.5-8 keV (full) X-ray bands and the source catalogs cross correlated, resulting in 265 unique sources detected in the two pointings of the $\mathrm{Cl} 1604$ system. Source properties, including count rates and detection significances, were determined with follow-up aperture photometry on all sources found by wavdetect. The photometry was carried out on the vignetting-corrected, soft- and hard-band images of both pointings and an appropriate aperture correction (as determined from the point-spead function (PSF) libraries in the Chandra calibration database) was applied to the background-subtracted net counts of each source. Counts in the full band were then determined as a sum of the measured net counts in the soft and hard bands. Of the 265 sources found by wavdetect, 161 had detection significances greater than $3 \sigma$ in at least one X-ray band.

Source fluxes in the soft and hard bands were determined by normalizing a power-law spectral model to the net count rate measured for each source. These rates were determined by dividing the net counts measured in the vignetting-corrected images by the nominal exposure time at the aim point of each observation. We assumed a photon index of $\gamma=1.4$ for the power-law model and a Galactic neutral hydrogen column density of $1.21 \times 10^{20} \mathrm{~cm}^{-2}$ (Dickey \& Lockman 1990), resulting in a count rate to unabsorbed-flux conversion factor of $5.85 \times 10^{-12}$ and $2.08 \times 10^{-11} \mathrm{erg} \mathrm{cm}^{-2} \mathrm{~s}^{-1}$ per

\footnotetext{
5 http://cxc.harvard.edu/
} 
counts $s^{-1}$ in the soft and hard bands, respectively. Full-band fluxes were again determined by summing the flux in the soft and hard bands. Rest frame X-ray luminosities were calculated for sources matched to galaxies with measured redshifts (see Section 3 ) using the standard luminosity distance equation and a $(1+z)^{\gamma-2} k$-correction. Properties for the 161 unique point sources detected with at least a $3 \sigma$ significance in the field of Cl1604 are listed in Table 3 of K09.

\subsection{Optical Imaging}

The optical imaging of the supercluster used for this study consists of 17 pointings of the Advanced Camera for Surveys (ACS) on the Hubble Space Telescope (HST). The ACS camera consists of two $2048 \times 4096$ CCDs with a pixel scale of 0 '.05 $^{\prime}$ pixel $^{-1}$, resulting in a $\sim 3^{\prime} \times 3^{\prime}$ field of view (FOV). The 17 pointing mosaic was designed to image nine of the ten galaxy density peaks observed in the field of the supercluster by $\mathrm{Gal}$ et al. $(2005,2008)$. Observations were taken in both the F606W and F814W bands and consist of 15 pointings from GO11003 (PI Lubin) with effective exposure times of $1998 \mathrm{~s}^{6}$ and 2 GTO pointings from G0-9919 (PI Ford) centered on clusters Cl1604+4304 and Cl1604+4321 with effective exposure time of $4840 \mathrm{~s}$. Our average integration time of $1998 \mathrm{~s}$ resulted in completeness depths of $\sim 26.5 \mathrm{mag}$ in each band.

The ACS data were reduced using the pipeline developed by the HST Archive Galaxy Gravitational Lens Survey (HAGGLES) team. The pipeline processing consists of calibrating the raw data using the best reference files provided by the HST archive, subtracting the background on each chip, iteratively determining the best shifts between the dithered exposures using multiple calls to SExtractor (Bertin \& Arnouts 1996) and multidrizzle (Koekemoer et al. 2002), aligning the final drizzled image to the USNO-B1 catalog (Monet et al. 2002) and resampling the images to a pixel scale of $0.03^{\prime \prime}$ pixel $^{-1}$. Object detection and photometry was carried out using SExtractor in dual-image mode, with the detection image being a weighted average of the F606W and F814W images. Detection parameters DETECT_MINAREA and DETECT_THRESH were set to three pixels above $3 \sigma$, while the deblending parameters DEBLEND_NTHRESH and DEBLEND_MINCONT were set to 32 and 0.03 , respectively. An outline of the region covered by the ACS mosaic relative to the ACIS-I observations is shown in Figure 1. Full details of the HAGGLES pipeline can be found in Marshall et al. (2009) and T. Schrabback et al. (2009, in preparation).

\subsection{Optical Spectroscopy}

The C11604 supercluster has been extensively mapped using the Low-Resolution Imaging Spectrograph (LRIS; Oke et al. 1995) and the Deep Imaging Multi-object Spectrograph (DEIMOS; Faber et al. 2003) on the Keck 10 m telescopes (Oke et al. 1998; Postman et al. 1998; Lubin et al. 1998; Gal \& Lubin 2004; G08). The complex target selection, spectral reduction, and redshift measurements are described in detail in Section 3 of G08. The final spectroscopic catalog contains 1618 unique objects. Redshifts derived for these objects are given a spectroscopic quality, $Q_{\text {spect }}$, between 1 and 4 , where 1 indicates that a secure redshift could not be determined due to poor signal, lack of features, or reduction artifacts, 2 is a redshift obtained from either a single feature or two marginally detected features,

\footnotetext{
6 With the exception of one pointing which lost guiding due to an incorrect
} attitude, resulting in a useable exposure time of $1505 \mathrm{~s}$ and a gap in the mosaic.

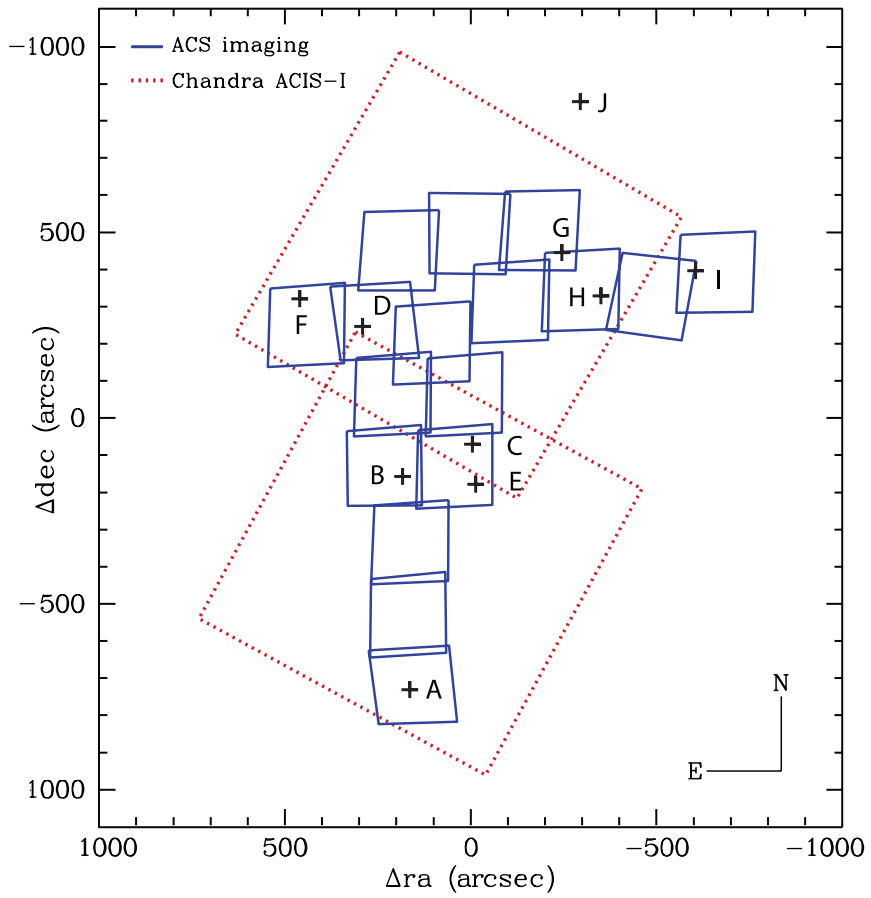

Figure 1. Positions and FOVs of the two ACIS-I and 17 ACS pointings in the Cl1604 region. The positions of the ten red-galaxy overdensities found by $\mathrm{Gal}$ et al. $(2005,2008)$ are marked and labeled following the naming convention of Gal et al. (2005).

(A color version of this figure is available in the online journal.)

3 is a redshift derived from at least one secure and one marginal feature, and 4 is assigned to spectra with redshifts obtained from several high signal-to-noise features. $Q_{\text {spect }}=-1$ is used for sources securely identified as stars.

In this sample, we find 140 stars and 1138 extragalactic objects with $Q_{\text {spect }} \geqslant 3$. A total of 417 galaxies are in the nominal redshift range of the supercluster between $0.84 \leqslant z \leqslant$ 0.96 . This extensive spectroscopic database is larger by a factor of $\sim 10$ than that for any other known moderate-to-high redshift supercluster.

\section{IDENTIFYING SUPERCLUSTER AGNs}

To find optical counterparts to the $161 \mathrm{X}$-ray point sources detected in the field of the $\mathrm{Cl} 1604$ supercluster we employed a maximum-likelihood technique described by Sutherland \& Saunders (1992) and more recently implemented by Taylor et al. (2005) and Gilmour et al. (2007). ${ }^{7}$ The method gauges the likelihood that a given optical object is matched to an Xray source by comparing the probability of finding a genuine counterpart with the positional offset and magnitude of the optical candidate relative to that of finding a similar object by chance. The advantages of this approach over a simple nearest neighbor match is that it takes into account the density of objects as faint as the optical counterpart as well as the distance between sources and the X-ray positional errors. The details of the process are described in K09 and therefore we focus instead on the results of the matching here.

Using the maximum-likelihood technique we have matched 125 optical sources to our X-ray catalog. Of these 42 have spectroscopic information available and we have derived reliable redshifts $\left(Q_{\text {spect }} \geqslant 3\right)$ for 35 of the sources. We find

\footnotetext{
7 See also Ciliegi et al. (2003), Brusa et al. (2005), and Mainieri et al. (2008).
} 
Table 1

X-ray Properties of X-ray-Selected AGNs in the C11604 Supercluster

\begin{tabular}{|c|c|c|c|c|c|c|c|}
\hline ID & Name & $\begin{array}{c}\text { R.A. } \\
\text { (J2000) }\end{array}$ & $\begin{array}{c}\text { Decl. } \\
(\mathrm{J} 2000)\end{array}$ & $z$ & $\begin{array}{l}\text { Net Counts } \\
(0.5-8 \mathrm{keV})^{\mathrm{a}}\end{array}$ & $\begin{array}{c}L_{\mathrm{x}}\left(\times 10^{43}\right) \\
(0.5-8 \mathrm{keV})^{\mathrm{b}}\end{array}$ & HR \\
\hline 0 & $\mathrm{~J} 160401.3+431351$ & 241.00546 & 43.23089 & 0.927 & 55.2 & 3.44 & $-0.31 \pm 0.17$ \\
\hline 2 & $\mathrm{~J} 160406.1+431807$ & 241.02528 & 43.30222 & 0.913 & 47.5 & 2.99 & $-0.36 \pm 0.19$ \\
\hline 3 & $\mathrm{~J} 160408.2+431736$ & 241.03416 & 43.29359 & 0.937 & 32.9 & 2.75 & $0.12 \pm 0.22$ \\
\hline 4 & $\mathrm{~J} 160410.9+432111$ & 241.04547 & 43.35318 & 0.935 & 10.3 & 1.12 & $0.52 \pm 0.53$ \\
\hline 6 & $\mathrm{~J} 160423.9+431125$ & 241.09959 & 43.19052 & 0.867 & 68.1 & 3.39 & $-0.42 \pm 0.16$ \\
\hline 7 & $\mathrm{~J} 160425.9+431245$ & 241.10783 & 43.21265 & 0.871 & 25.9 & 1.31 & $-0.42 \pm 0.28$ \\
\hline 8 & $\mathrm{~J} 160436.7+432141$ & 241.15290 & 43.36147 & 0.923 & 29.9 & 2.28 & $-0.10 \pm 0.24$ \\
\hline
\end{tabular}

Notes.

a Net counts corrected for vignetting.

b In units of $h_{70}^{-2} \mathrm{erg} \mathrm{s}^{-1}$.

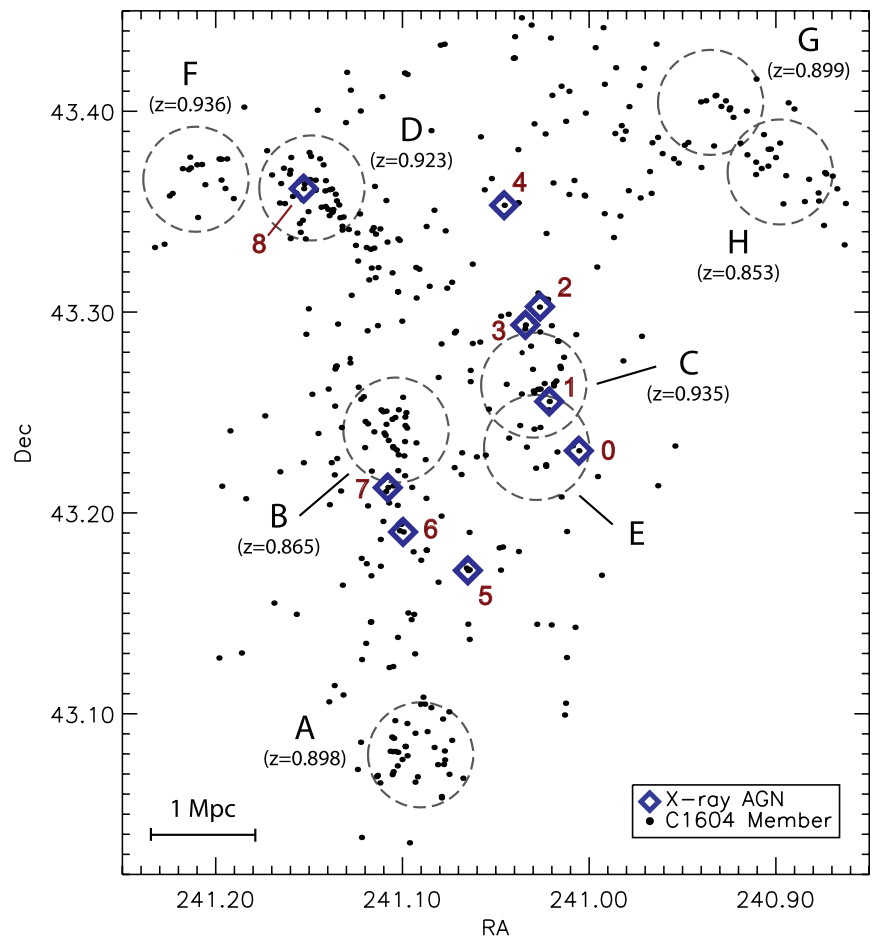

Figure 2. Spatial distribution of the nine $X$-ray sources that fall within the traditional redshift boundaries of the $\mathrm{Cl1604}$ supercluster $(0.84<z<0.96)$. Individual clusters and groups in the $\mathrm{Cl1604}$ system are labeled using the naming convention of Gal et al. (2005). The dashed circles extend $0.5 h_{70}^{-1} \mathrm{Mpc}$ from the center of each cluster.

(A color version of this figure is available in the online journal.)

that while the matched sources cover a wide range of redshifts $(0.055 \leqslant z \leqslant 2.30)$, the distribution exhibits a clear peak at $z=0.9$, the average redshift of the C11604 supercluster (see Figure 6 of K09). A total of nine sources have redshifts between $0.84<z<0.96$ and fall within the redshift boundaries of the supercluster. ${ }^{8}$ Eight of these nine galaxies were imaged by our ACS survey of the supercluster and therefore have highresolution imaging available for morphological and resolved color analyses. The coordinates and redshifts of the nine supercluster AGNs are listed in Table 1 and their location in the supercluster are shown in Figure 2.

\footnotetext{
8 Eight of the redshifts were derived with $Q_{\text {spect }}=4$ and one source, no. 2 in
} Table 1 , has a $Q_{\text {spect }}=3$ redshift.
Also listed in Table 1 are the X-ray properties of the nine AGNs, including their rest-frame $0.5-8 \mathrm{keV}$ luminosities and hardness ratios (HRs), measured as $\mathrm{HR}=\frac{H-S}{H+S}$ where $H$ and $S$ are the net counts in the hard and soft bands, respectively. The AGNs have luminosities ranging from 0.78 to $4.47 \times 10^{43} h_{70}^{-2} \mathrm{erg} \mathrm{s}^{-1}$ and have predominantly soft $\mathrm{X}$ ray spectra; the nine sources have a median $\mathrm{HR}$ of -0.20 . It should be noted that the luminosity of all nine AGNs exceed the luminosity attributable to starburst galaxies by over an order of magnitude $\left(L_{\mathrm{X}} \sim 10^{42} \mathrm{erg} \mathrm{s}^{-1}\right.$; e.g., Bauer et al. 2002), making it highly unlikely that their X-ray emission is powered exclusively by star formation activity. On the other hand, their luminosities fall below the level of bright QSOs $\left(L_{\mathrm{X}} \sim 10^{44} \mathrm{erg} \mathrm{s}^{-1}\right)$, which often dominate the optical emission from their host galaxies. Silverman et al. (2005) have shown that the optical emission associated with AGNs with luminosities below $5 \times 10^{43} h_{70}^{-2} \operatorname{erg~s}^{-1}(0.5-8 \mathrm{keV})$ is primarily due to light from the host galaxy. Since the luminosity of all our AGNs fall below this limit, we proceed under the assumption that the optical properties of the nine supercluster host galaxies suffer minimal contamination from their active nuclei.

\section{AGN HOST PROPERTIES}

\subsection{Morphologies}

To gain insight into the possible triggering mechanisms of the X-ray-detected AGN found in the Cl1604 supercluster we examined the morphologies of the host galaxies within which they reside using both visual and automated techniques. We limited this analysis to the eight AGNs which are covered by our high-resolution ACS imaging. Thumbnail images in the F814W band of each supercluster AGN are show in Figures 3 and 4.

Classification of the host morphologies was carried out via visual inspection by two of the authors (L.M.L. and C.D.F.). The resulting classifications are listed in Table 2. Also noted are the interpretations of any observed disturbances using the classification scheme of Smail et al. (1997). We find that a large majority (7/8) of the host galaxies are spheroidal systems. Five of the supercluster AGN reside in early-type galaxies (E/S0), two hosts are bulge dominated systems with faint spiral arms (Sa), and only a single host is a clear disk dominated, late-type galaxy $(\mathrm{Sb})$.

These visual classifications were confirmed by the calculation of the Gini coefficient, $G$, which is a measure of the symmetry in a galaxy's flux distribution (Abraham et al. 2003) and the $M_{20}$ 

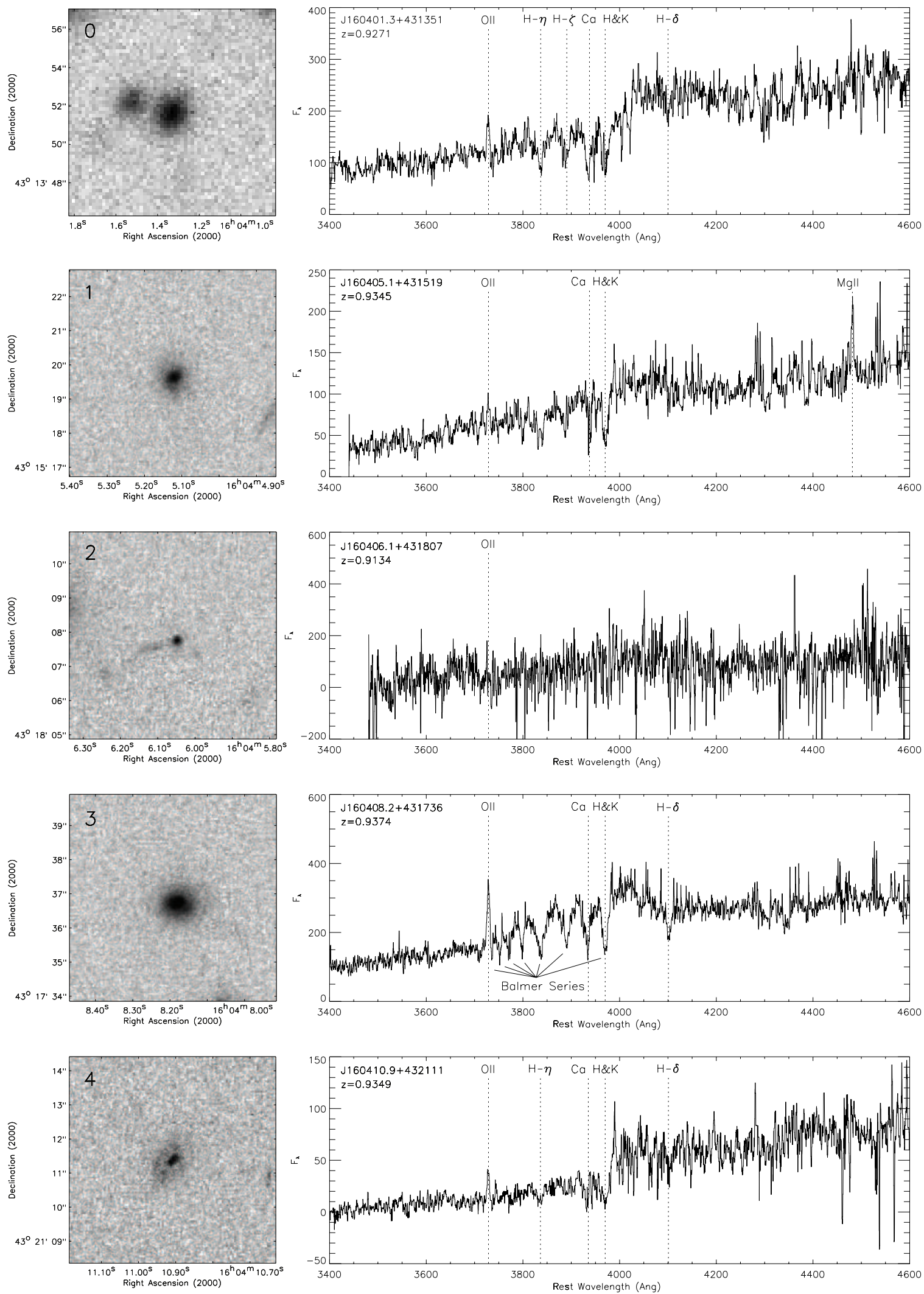

Figure 3. Left: thumbnail images of the eight supercluster AGNs imaged with HST/ACS in the F814W band as well as one source imaged with Palomar/LFC in the $i^{\prime}$ band. Each image spans roughly 6" on a side and is shown with a log scaling. Right: optical spectra of all nine supercluster AGNs obtained with Keck/DEIMOS. The flux scale is presented in self-consistent, arbitrary units. The spectra have typically been binned by 10 pixels for clarity, where one pixel equals $0.33 \AA$.

parameter, which is the second-order moment of the brightest $20 \%$ of a galaxy's flux and a measure of central concentration (Lotz et al. 2004). We measured the parameters using software written by one of the authors (R.L.) and described in Lin (2007). We find that although our absolute scaling of both $G$ and $M_{20}$ differ from that of other software used in the literature (due 

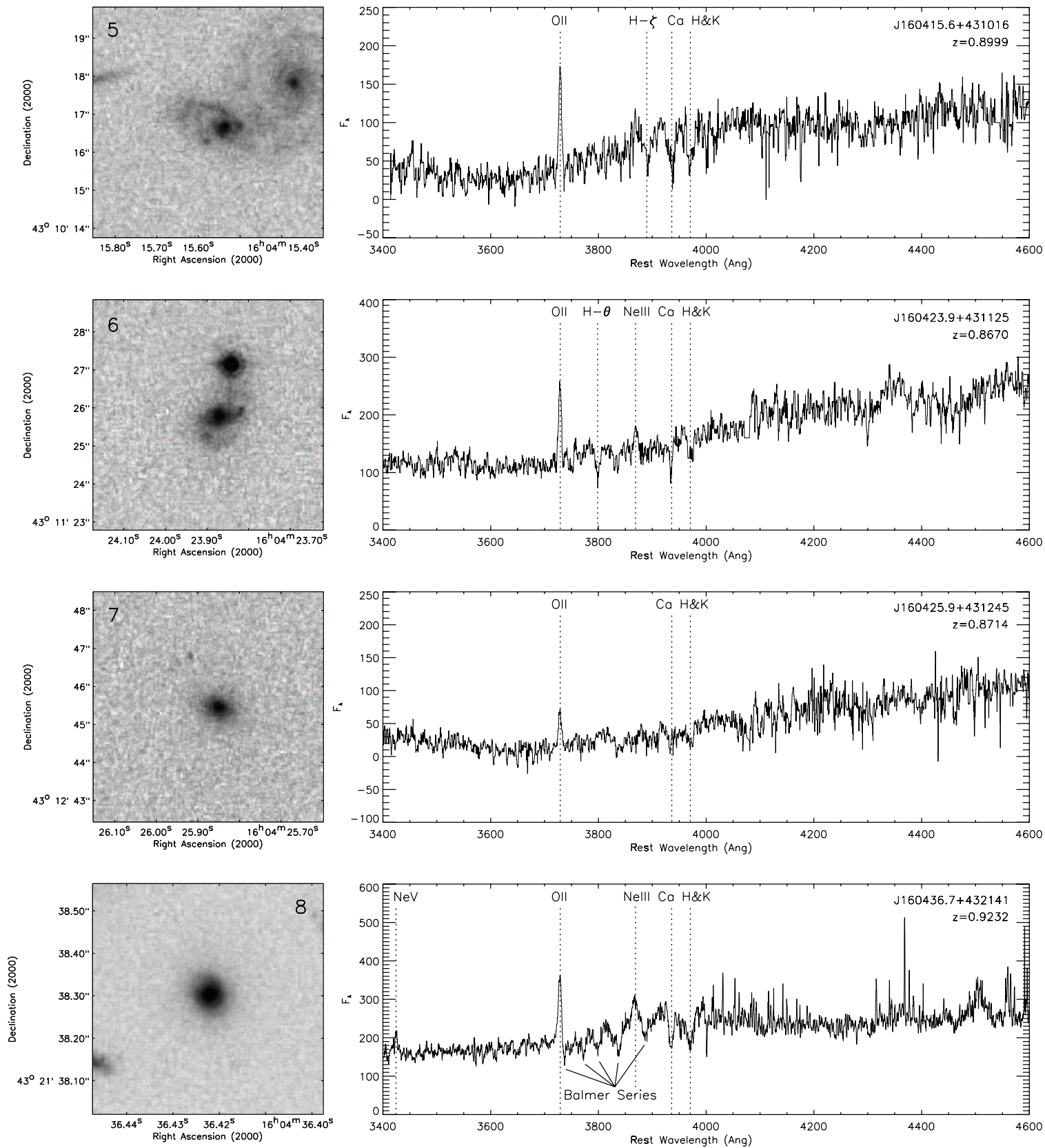

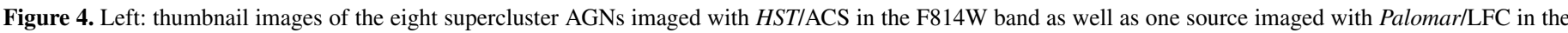

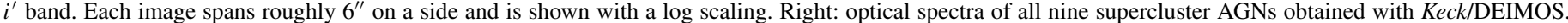
The flux scale is presented in self-consistent, arbitrary units. The spectra have typically been binned by 10 pixels for clarity, where one pixel equals $0.33 \AA$.

to a different implementation and measurement aperture, see Lisker 2008), general trends remain, with spheroids at lower $M_{20}$ values than disk-dominated systems and both being well separated from merger systems which have higher $G$ values for a given $M_{20}$ range.

The location of the eight host galaxies in the $G-M_{20}$ parameter space is shown in Figure 5. We again find that the supercluster AGNs largely favor early-type hosts. We find that 5 of 8 host galaxies have parameters consistent with spheroidal and bulge dominated systems (E/SO/Sa), one source (no. 4) lies in the intermediate region occupied by $\mathrm{Sb} / \mathrm{bc}$ galaxies, consistent with its visual classification as a spiral galaxy, and two sources fall within the merger regime. The two merger galaxies are sources 5 and 6, which have bright nearby companions. While it remains uncertain whether the companion to source 6 is simply seen in projection, spectroscopy has confirmed that source 5 and its spiral companion are indeed a kinematic pair.

Although only two galaxies fall within the merger regime of the $G-M_{20}$ plane, we find indications of merger activity and/or recent interactions in many of the hosts. This is most evident in source 2, which appears to be a merger remnant with a very compact core and faint tidal features. We suspect the flux contribution from the tidal features was insufficient to alter the galaxy's symmetry and concentration enough to move it into the merger regime of the $G-M_{20}$ parameter space. Nonetheless, the multiple faint (and blue) tidal features surrounding the source suggests that the system underwent a disruptive merger event in the recent past. Faint tidal features are also seen around source 6 which has a nearby point-like companion. Furthermore, we find that source 0 , which falls outside of our ACS mosaic, also shows 
Table 2

Optical Properties of X-ray-Selected AGNs in the C11604 Supercluster

\begin{tabular}{lccccccc}
\hline \hline ID & Name & $\begin{array}{c}\text { [O II }] \text { EW } \\
(\AA)\end{array}$ & $\begin{array}{c}\mathrm{H} \delta \text { EW } \\
(\AA)\end{array}$ & $\begin{array}{c}\text { SFR } \\
\left(M_{\odot} \mathrm{yr}^{-1}\right)\end{array}$ & $\begin{array}{c}\text { Morph } \\
\text { Class }\end{array}$ & $\begin{array}{c}\text { Int }^{\mathrm{b}} \\
\text { Notes }\end{array}$ \\
\hline 0 & $\mathrm{~J} 160401.3+431351$ & -3.3 & 1.8 & $2.7 \pm 1.3$ & $\ldots$ & $\ldots$ & No ACS data available \\
1 & $\mathrm{~J} 160405.1+431519$ & -2.5 & $\ldots$ & $1.6 \pm 0.9$ & $\mathrm{E}$ & $\ldots$ & Blue core, faint elongation, possible tidal feature \\
2 & $\mathrm{~J} 160406.1+431807$ & -2.0 & $\ldots$ & $0.6 \pm 0.5$ & $\mathrm{E} / \mathrm{X}$ & $\mathrm{T}$ & Merger remnant, tidal features \\
3 & $\mathrm{~J} 160408.2+431736$ & -7.2 & 5.2 & $7.7 \pm 3.5$ & Epec & $\mathrm{M}$ & Blue, double peaked core \\
4 & $\mathrm{~J} 160410.9+432111$ & -5.2 & 6.0 & $0.9 \pm 0.6$ & Sa & $\ldots$ & Red galaxy with elongated core \\
5 & $\mathrm{~J} 160415.6+431016$ & -16.8 & $\ldots$ & $4.5 \pm 2.1$ & Sb & $\mathrm{M}$ & Spiral with kinematic companion \\
6 & $\mathrm{~J} 160423.9+431125$ & -7.5 & $\ldots$ & $4.3 \pm 2.0$ & Sa & $\mathrm{M}$ & Blue core, nearby companion, tidal features \\
7 & $\mathrm{~J} 160425.9+431245$ & -13.7 & $\ldots$ & $1.3 \pm 0.7$ & $\mathrm{E}$ & $\ldots$ & Blue core, appears relaxed \\
8 & $\mathrm{~J} 160436.7+432141$ & -7.0 & $\ldots$ & $8.5 \pm 3.9$ & $\mathrm{E}$ & $\ldots$ & \\
\hline
\end{tabular}

Notes.

a The standard Hubble classification scheme (E, S0, Sa, Sb, . .) with the addition of the following: X, compact object; pec, peculiar.

${ }^{\mathrm{b}}$ Interpretation of disturbance: T, tidal feature; $\mathrm{M}$, merger.

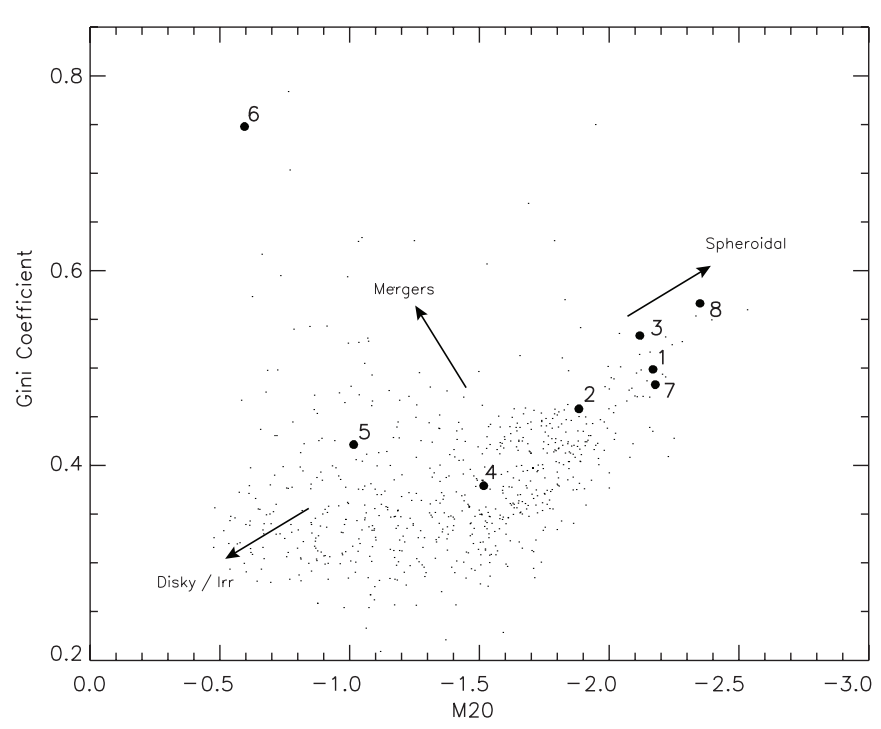

Figure 5. Gini and $M_{20}$ parameters for the eight supercluster AGNs imaged with $H S T /$ ACS along with a subsample of normal galaxies in the same field. We find that the AGN hosts are predominantly early-type or bulge-dominated galaxies.

evidence of a nearby companion in our ground-based imaging. An image of this galaxy taken with the Large Format Camera (LFC) on Palomar in the Sloan Digital Sky Survey (SDSS) i' filter is shown in Figure 3; details of our ground-based imaging and its reduction are described in Gal et al. (2005).

Other merger indications include double and/or elongated cores (see Figure 6). Source 3 has a partially resolved double core separated by 0.13 in projection or $\sim 1.0 h_{70}^{-1} \mathrm{kpc}$ at $z=$ 0.937 . This separation is similar to the $z=0.709$ dual AGNs reported by Gerke et al. (2007) which has a separation of roughly $1.2 h_{70}^{-1} \mathrm{kpc}$, although here we do not resolve spectral features from multiple active nuclei. Despite the presence of multiple cores, the outer portions of the galaxy appear fairly relaxed with no signs of tidal features or significant disruptions. A twodimension surface brightness fit using the GALFIT software (Peng et al. 2002) found the galaxy's outer profile to be well fit by a Sersic index (Sersic 1968) of $n=3.1$, close to a standard de Vaucouleurs profile (de Vaucouleurs 1948). This suggests that if the galaxy is the result of a major merger, sufficient time has elapsed since the event to render the galaxy largely relaxed. Source 4 also shows signs of an elongated core, although here we do not resolve multiple components. The cores of both source 3 and 4 are shown in Figure 6.

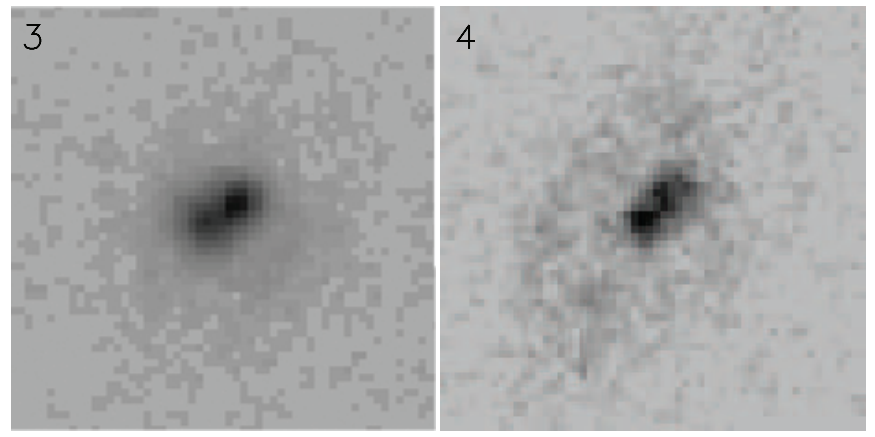

Figure 6. $H S T /$ ACS images of Source 3 (left) and Source 4 (right) in the F814W band, with scaling appropriate to highlight the double and/or elongated cores present in each galaxy.

In summary, our visual morphological classifications and those indicated by the $G$ and $M_{20}$ parameters suggest that the AGNs in Cl1604 supercluster largely favor early-type and bulgedominated host galaxies. We also find that a majority (6/9) show signs of recent or pending interactions, with one AGN in a clear merger remnant, three with nearby companions, and an additional two with disrupted cores, despite the fact that only two of these are identified as bona fide mergers by their $G$ and $M_{20}$ parameters alone. Our results are in good agreement with the well-established finding that AGNs are predominantly hosted by early-type galaxies (e.g., Kauffmann et al. 2003), although we do observe a larger fraction of disturbed morphologies than was reported by Grogin et al. (2005) and Pierce et al. (2007), who found little evidence for recent mergers in the hosts highredshift AGN. We discuss this disparity in greater depth in Section 6.

\subsection{Colors}

The optical colors and magnitudes of the 371 supercluster members observed with ACS in the F606W and F814W bands are show in Figure 7. The color bimodality of the galaxies is clearly apparent, with members largely separated into the supercluster red sequence and a more diffuse blue cloud. The presence of such a large number of supercluster members in the blue cloud is a classic example of the Butcher-Oemler effect (Butcher \& Oemler 1984), where higher redshift clusters are observed to have an increased fraction of blue, star-forming galaxies relative to the passive cluster populations observed at low redshift. 


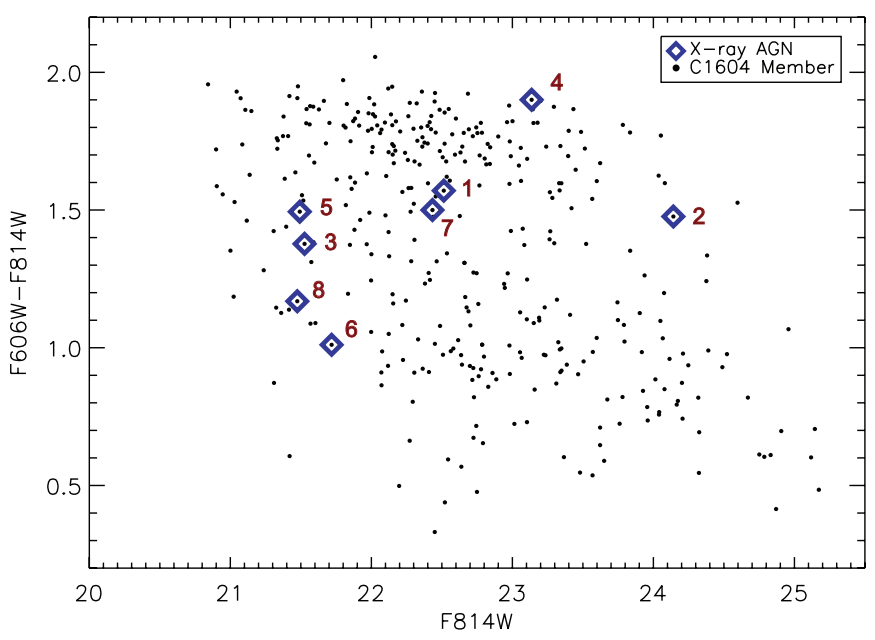

Figure 7. Optical color (F606W-F814W) as a function of apparent magnitude in the F814W band for the eight supercluster AGNs imaged with HST/ACS. Also shown are the colors of 363 normal supercluster members.

(A color version of this figure is available in the online journal.)

Also highlighted in Figure 7 are the colors of the eight AGN hosts imaged with ACS. We find that with the exception of source 4, all of the host galaxies are bluer than the red sequence. That said, only two sources are clearly in the blue cloud. Depending on where one defines the boundary of the blue cloud, we find that 4-5 of 8 hosts have intermediate colors between the red and blue populations and are located in the transition zone of the CMD. Regarding the reddest host, source 4, the galaxy is redder than the supercluster red-sequence and has the hardest $\mathrm{X}$-ray emission of the nine AGNs, with HR $=0.52$. This is indicative of heavy obscuration, suggesting that the galaxy's color is most likely due to heavy extinction rather than a passive stellar population. The high fraction of hosts we find in the transition zone of the CMD is in excellent agreement with the results of Silverman et al. (2008), who recently reported an increased fraction of hosts with intermediate colors in a narrow redshift window containing two large-scale structures in the E-CDF-S.

Also apparent in Figure 7 is that six of eight AGNs ( 75\%) are located in luminous hosts with $m_{814}<23$. This translates to a rest frame $M_{V}=-21.1$ at $z=0.9$ using a $k$-correction appropriate for an $\mathrm{Sb}$ galaxy spectrum. The preference for AGNs to be located in optically luminous host galaxies has been previously reported in low-redshift superclusters (Gilmour et al. 2007) and in the field over a wide range of redshifts (Barger et al. 2003; Nandra et al. 2007; Silverman et al. 2008). Silverman et al. (2008) found that $80 \%$ of the moderate-luminosity AGNs in their sample reside in galaxies with $M_{V}<-20.7$, in excellent agreement with our results, despite our small number statistics.

Our high-resolution, dual band ACS observations also allow us to resolve color gradients in the AGN host galaxies in order to investigate any recent star formation activity. These gradients are shown in Figure 8, along with color thumbnails of each host constructed from observations in the F814W and F606W bands. We find that four sources exhibit blue cores similar to those reported by Menanteau et al. (2001a) and Treu et al. (2005). These include source 1 , the double-cored source 3 , source 6 which has a nearby companion and the fairly undisturbed source 8 .

These blue cores may be due to contamination by scattered AGN emission, although we find this unlikely as (1) the
X-ray luminosity of our sources does not reach the QSO level $\left(L_{X} \sim 10^{44} \mathrm{erg} \mathrm{s}^{-1}\right)$ and $(2)$ the spectra of these four sources show no signs of a rising blue continuum (see Section 4.3) indicative of QSO contamination. The blue cores are more likely the result of nuclear and/or circumnuclear star formation predicted from merger simulations (Mihos \& Hernquist 1996) and observed in low redshift AGNs (e.g., Gu et al. 2001). In fact, Menanteau et al. (2001b) find that a merger induced episode of star formation responsible for producing as little as $20 \%$ of the total stellar mass of the system can lead to blue cores in otherwise red spheroids similar to the galaxies found by Menanteau et al. (2001a) and Treu et al. (2005). The formation mechanism proposed by Menanteau et al. (2001b) is consistent with our observations as three of the sources have fairly red (F606W-F814W 1.7-1.8) colors outside their centers. We discuss the implications of this observation further in Section 6.

\subsection{Spectral Properties}

Spectra of all nine supercluster AGN hosts obtained with the DEIMOS spectrograph are shown in Figures 3 and 4. We find that all of the hosts exhibit narrow [O II] emission lines of low to moderate strength. ${ }^{9}$ The equivalent widths (EW) of these lines, which were measured using the bandpass definitions of Fisher et al. (1998), are listed in Table 2; the values range from -2.0 to $-16.8 \AA$. We also observe that a majority (six of eight) show Balmer absorption features indicative of young A-type stars and the lack of massive O- and B-type stars. Two hosts (nos. 3 and 4) show $\mathrm{H} \delta$ absorption lines with EWs of 5.2 and $6.0 \AA$, respectively, while a second pair (nos. 0 and 8 ) exhibit a strong higher-order Balmer series. The most dramatic sets of Balmer lines are seen in sources 3 and 8, two of the blue-cored, early-type hosts. We note that none of the sources show signs of broad line emission or significantly rising blue continua commonly found in more luminous QSOs, suggesting little AGN contamination at shorter wavelengths.

If not for the observed [O II] emission lines, many of the host galaxies would be classified as post-starburst due to their Balmer absorption. Balmer lines typically persist for up to $\sim 1.0 \mathrm{Gyr}$ after the last major episode of star formation due to the lifetime of A-type stars (Poggianti \& Barbaro 1997) and are usually not visible in systems with high levels of ongoing star formation due to emission filling from $\mathrm{H}$ in regions. Unfortunately, given our spectral window we cannot use other diagnostic lines such as $\mathrm{H} \alpha$ to determine whether the [O II] features are due to ongoing star formation or photoionization by the AGN. If we assume the [O II] emission is due entirely to star formation, then converting the $[\mathrm{O} \mathrm{II}]$ line luminosities to star formation rates (SFRs) using the relationship of Kennicutt (1998) we obtain rates ranging from $0.6 \pm 0.5$ to $8.5 \pm 3.9 M_{\odot} \mathrm{yr}^{-1}$. All of the derived rates are listed in Table 2 and represent lower limits as they are not corrected for extinction.

While it is more than feasible that many of these galaxies have some ongoing, centrally concentrated star formation given their bluer colors, our calculated rates are likely overestimated (barring extinction corrections) as it is distinctly possible that AGN-related emission contributes to the [O II] line luminosity. This was recently demonstrated by Yan et al. (2006), who found that post-starburst galaxies in the SDSS sample which show

\footnotetext{
9 Despite the low signal to noise of the source 2 spectrum and a problematic background subtraction, we do observe a double-peaked emission line indicative of [O II] at $z=0.9134$ in the extracted spectrum of this host. A two-dimensional spectrum of the galaxy is shown in Figure 9 with these [O II] emission features marked.
} 

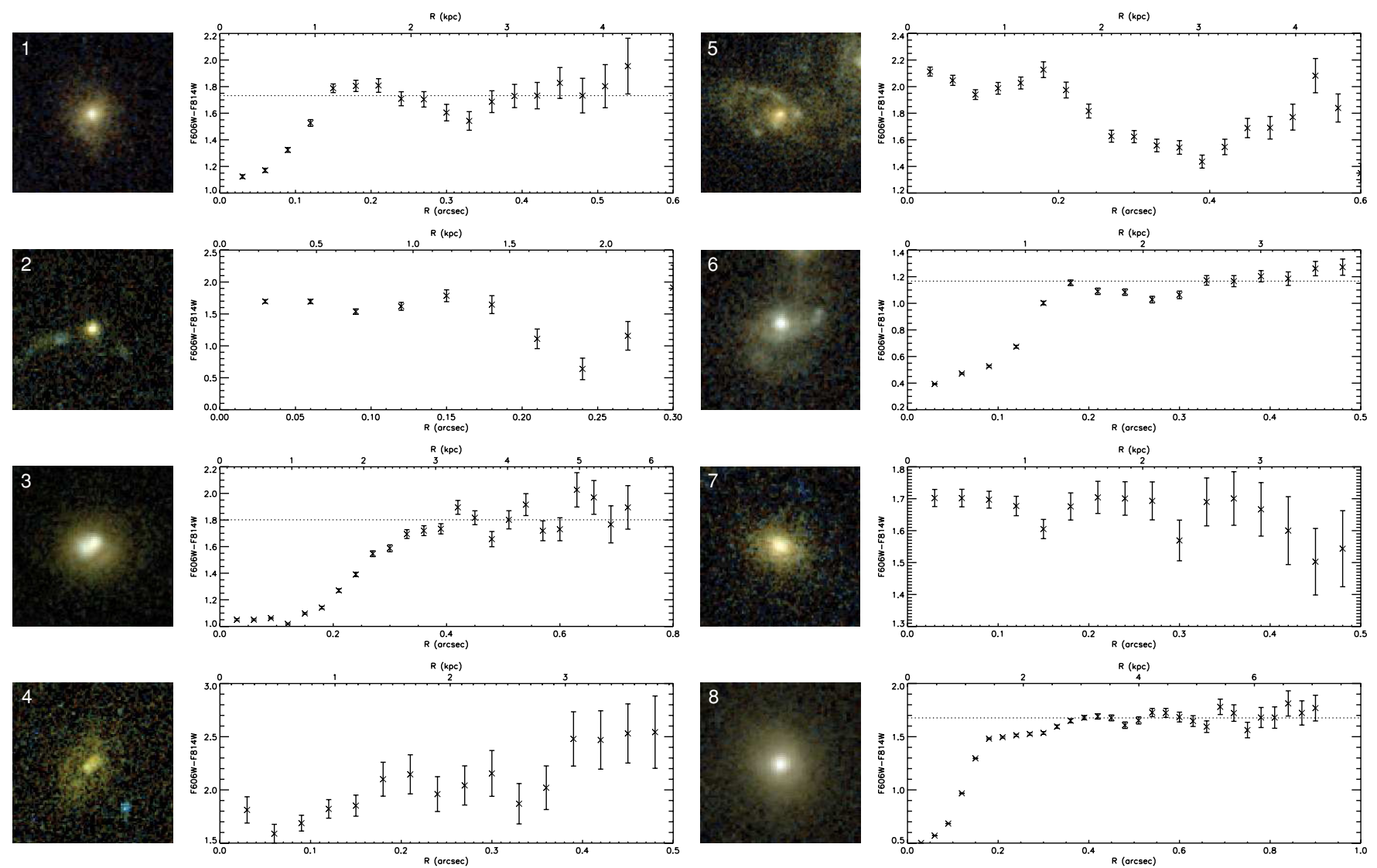

Figure 8. Left: color thumbnail images of the eight supercluster AGNs imaged with HST/ACS. The color components are F606W and F814W for blue and red, respectively, while green is represented by a weighted average of the two. The images sizes range between 2 '.5 and 3'.5 on a side. Right: spatially resolved color profiles (F606W-F814W) of each AGN host. The profiles are centered on the galaxy nucleus and represents the median galaxy color in annuli one pixel wide.

little (or no) $\mathrm{H} \alpha$ emission, but have line ratios consistent with Seyferts, often exhibit significant [O II] EWs. We have recently undertaken near-infrared spectroscopy of a small subsample of Cl1604 members using NIRSPEC on Keck to determine $\mathrm{H} \alpha$ line strengths in order to perform a similar test at $z=0.9$. Of the X-ray sources discussed in this study, only source 5 has thus far been observed. This galaxy has the highest measured [O II] EW of the nine hosts and we find an $\left[\mathrm{N}_{\mathrm{II}}\right] / \mathrm{H} \alpha$ line ratio consistent with the system being a transition object, which suggests that both star formation and AGN emission contribute to the system's [O II] line flux. Therefore, the [O II]-derived SFR for this galaxy is likely an overestimate as it does not account for the AGN contribution. We plan to observe the remaining eight sources, including the four post-starburst hosts, with NIRSPEC in the near future.

It is worth noting that the highest measured SFRs, which are $7.7 \pm 3.5$ and $8.5 \pm 3.9 M_{\odot} \mathrm{yr}^{-1}$, are found in sources 3 and 8, respectively; the two galaxies with the most pronounced Balmer absorption. It is unlikely that these absorption lines would be so prevalent in systems that are forming stars as prodigiously as these rates suggest. Poggianti et al. (1999) found that spectra with strong Balmer lines and measurable [O II] can be reproduced with low-level star formation following a recent, strong burst. This may in fact be what we are observing here if our [O II]-derived SFRs are overestimated due to AGN contamination. Alternatively, the [O $\mathrm{OI}]$ emission may originate entirely from gas ionized by the AGN. In either case, it would seem that a large fraction of our supercluster AGNs are located in galaxies which underwent a starburst phase within the last $\sim 1$ Gyr and whose star formation has since been truncated.
In addition to the post-starburst features, most hosts also show $\mathrm{Ca} \mathrm{H} \& \mathrm{~K}$ lines and three sources have a clear continuum break at $4000 \AA$, features indicative of an underlying older stellar population. This is observed in the spectra of sources 3,6 , and 8 , all of which are blue-cored galaxies with otherwise red colors. This set of observed properties is consistent with a minor merger induced nuclear starburst in a previously passive system, similar to the process described by Menanteau et al. (2001b).

\subsection{Environments}

The location of the nine AGNs detected in the Cl1604 supercluster in relation to the system's constituent clusters are shown in Figure 2. We find the greatest concentration of AGN near Cl1604+4316 (hereafter Cluster C) at $z=0.935$. Four sources have redshifts nearly equal to the cluster's systemic redshift (nos. $0,1,3,4$ ), while a single source is located in the foreground of the cluster (no. 2 at $z=0.913$ ). Three of the AGNs are located on the outskirts of Cl1604+4314 (Cluster B) at $z=0.865$; the two sources closest to the cluster center have nearly identical redshifts to that of the cluster (nos. 6 and 7), while the one with the greatest projected distance is at a higher redshift (no. 5 at $z=0.899$ ). In Cl1604+4321 (Cluster D) we find a single AGN (no. 8) with a redshift identical to that of the systemic cluster redshift of $z=0.923$.

A striking result is the general absence of AGNs in regions with the highest local galaxy densities. This includes Cl1604+4304 (Cluster A) and the center of Cluster B. These systems are the richest clusters in the Cl1604 complex, with the highest measured velocity dispersions and X-ray luminosities (G08; K09). On the other hand, we do find AGN near the 


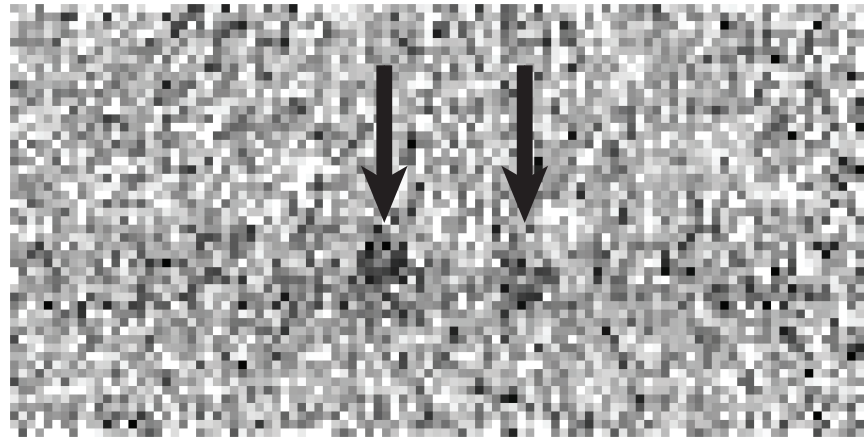

Figure 9. Two-dimensional optical spectrum obtained with Keck/DEIMOS of Source 2 near the [O II] doublet. [O II] emission features at 3726 and $3729 \AA$ are marked. The image has a pixel scale of $0.33 \AA$ pixel $^{-1}$ along the dispersion axis.

centers of Clusters C and D (both in projection and redshift space), both of which are poorer systems that are not directly detected in our X-ray observations. It should be noted that although the diffuse X-ray emission from Clusters A and B effectively increases our flux limit for point source detection near these clusters, the emission is of such a low level ( 73 counts within $50^{\prime \prime}$ of Cluster B, for example), that we are confident that any embedded point sources in this region would have been detected by visual inspection if not by wavdetect. In addition, in K09 we calculate the ACIS-I flux limit as a function of position over the entire $\mathrm{Cl} 1604$ field and find that all nine of the spectroscopically confirmed supercluster AGNs would have been detected if they had instead been located within the diffuse emission of Cluster A or B. Therefore, we do not believe this emission will significantly bias our analysis of the AGN environments.

The distribution of AGNs in the supercluster suggests their that activity may be influenced by both local environment and more large-scale cluster properties such as a system's dynamical state. This is highlighted by the presence of AGNs on the outskirts of Cluster B and the lack of a similar population around Cluster A. While both systems are relatively massive, G08 found evidence of velocity segregation in the redshift distribution of Cluster B, indicating the system may have undergone a recent merger or is actively accreting a substantial number of galaxies. K09 also found the system to have a higher than expected velocity dispersion relative to the $L_{\mathrm{x}}-\sigma_{v}$ relation, also suggesting that the system may not be fully relaxed. This is quite unlike Cluster A, which shows no signs of recent merger activity and appears fairly relaxed (and consistent with the $L_{\mathrm{x}}-\sigma_{v}$ relation). We speculate that the difference in the dynamical activity of Clusters A and B may indicate the global properties of these systems are affecting the level of AGN activity observed around each cluster.

In order to quantify the local environments of the AGN and to compare them to those of the entire supercluster population we calculated the local projected galaxy density, $\Sigma$, near each host galaxy using the method employed by Dressler (1980) and more recently by Smith et al. (2005) and Postman et al. (2005). The method calculates the projected density as $\Sigma=N / \Omega_{N}$, where $\Omega_{N}$ is the solid angle which encompasses a galaxy's $N$ nearest neighbors down to a certain absolute magnitude limit. For our calculation we adopt the parameters used by Postman et al. (2005) with $N=7$ and an absolute $V$-band magnitude of $M_{V}=-19.27$. At $z=0.9$, the average redshift of the supercluster, and using an $\mathrm{Sb}$ galaxy $k$-correction, the latter translates to an observed magnitude of $m_{814}=24.82$. We found

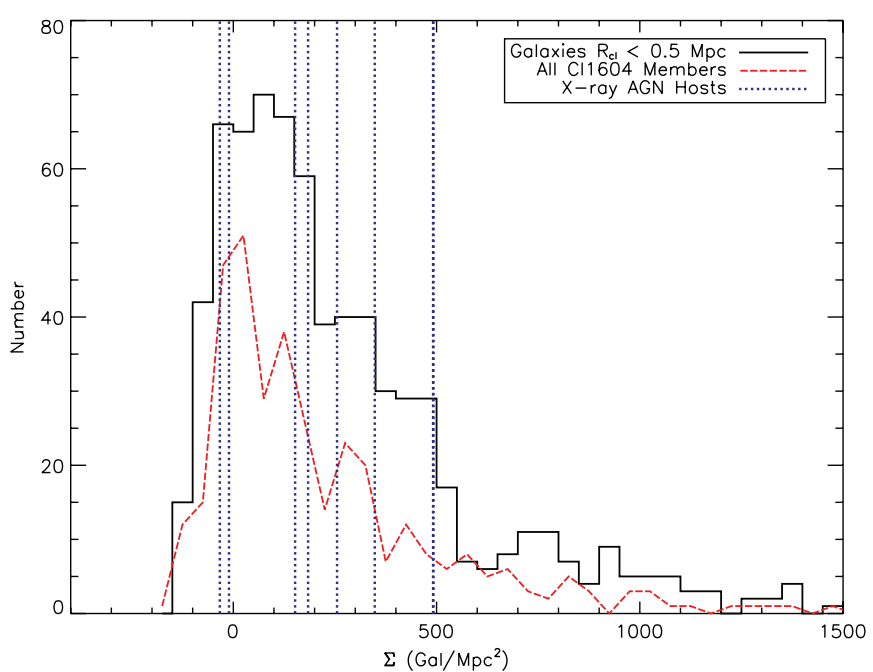

Figure 10. Projected galaxy density above the field density in the direction of the C11604 Supercluster. Densities were calculated using all galaxies down to $M_{V}=-19.27$. The density distribution for supercluster members and X-ray AGN hosts are denoted by the dashed red and vertical dotted blue lines, respectively. The solid histogram shows the density of all ACS detected galaxies within $0.5 h_{70}^{-1} \mathrm{Mpc}$ of a cluster center.

(A color version of this figure is available in the online journal.)

that varying these parameters in the range of $5 \leqslant N \leqslant 10$ and $24 \leqslant m_{814} \leqslant 25$ did not significantly alter our results.

Since the Cl1604 field lacks sufficient redshift information to calculate $\Sigma$ using only confirmed supercluster members, we make use of all galaxies falling above our magnitude limit to determine the projected density and statistically correct for background and foreground contamination. This correction is derived from the average surface density of field galaxies with $m_{814} \leqslant 24.82$ in seven blank fields observed with ACS. These fields were chosen for their lack of cataloged structures and include three fields from GO-9761 (P.I.: Alamini) and four fields from GO-9468 (P.I.: R. Yan). ${ }^{10}$ Each field was obtained from the HST archive and reduced in an identical manner to that of the Cl1604 data set. Since we correct for the background and foreground contamination by subtracting the average surface density of a blank field our projected densities differ from those of Smith et al. (2005) and Postman et al. (2005) in that they are technically densities in excess to the field densities. In other words, our projected densities have been normalized to the field density at $z=0.9$.

The distribution of projected densities for all 371 supercluster members imaged by ACS is shown in Figure 10. Also shown are the local densities of the eight AGN host galaxies. We find two sources (nos. 4 and 5) have densities similar to that of the field, which given our statistical correction is set to $\Sigma=0$ galaxies $\mathrm{Mpc}^{-2}$. The remaining sources are located in regions of intermediate density, with $\Sigma$ values larger than the field value, but well below the densities found near the cluster centers which range from 700 to 800 galaxies $\mathrm{Mpc}^{-2}$ in the center of Clusters C and D and $\sim 1000$ galaxies $\mathrm{Mpc}^{-2}$ in Clusters $\mathrm{A}$ and $\mathrm{B}$. These densities confirm our conclusions determined by visual inspection: the AGNs are found in intermediate density environments, either outside the centers of massive clusters or in poorer clusters. It has long been thought that such

\footnotetext{
10 The field centers are 03:42:48.37 -09:36:51.9, 09:54:34.40 +17:53:11.2, $10: 01: 46.32+55: 05: 24.7,16: 36: 58.01+40: 56: 01.1,16: 37: 04.11+40: 58: 59.1$, $16: 37: 04.11+41: 01: 58.1,21: 55: 42.97-09: 26: 34.7$.
} 
regions are conducive to galaxy interactions due to their higher densities compared to the field and their relatively low velocity dispersions (Cavaliere et al. 1992). This finding is similar to the conclusions of Gilmour et al. (2007) who found that AGNs in the A901/902 supercluster at $z \sim 0.17$ avoid the densest environments in the system and are instead located on the outskirts of clusters or in galaxy groups. At higher redshifts, we also agree with the results of Georgakakis et al. (2007) who found that AGNs generally avoid underdense regions and are found in richer environments than similar nonactive galaxies.

\section{CLUSTER AGN FRACTION}

There are several lines of evidence that suggest that AGN activity is triggered more often in and around galaxy clusters than in the field. A growing number of observations have reported an overdensity of X-ray point sources in the vicinity of clusters relative to blank-field observations (Henry \& Briel 1991; Cappi et al. 2001; Ruderman \& Ebeling 2005; K09) and there are indications this overdensity increases with redshift (Cappelluti et al. 2005; Branchesi et al. 2007). This trend was recently confirmed spectroscopically by Eastman et al. (2007), who found an order-of-magnitude increase in the number of cluster members hosting AGNs in four $z \sim 0.6$ clusters compared to the fraction measured by Martini et al. (2006) in eight low-redshift clusters $(z<0.31)$ using the same methodology. We can perform a similar analysis using the AGN in the $\mathrm{Cl1604}$ supercluster to investigate whether this trend continues out to $z \sim 0.9$.

Eastman et al. (2007) found a total of eight galaxies hosting AGNs with X-ray luminosities of $L_{\mathrm{X}}>10^{43} h_{70}^{-2} \mathrm{erg} \mathrm{s}{ }^{-1}$ (2-10 keV) within the $R_{200}$ radius of four higher-redshift clusters. This was out of $\sim 400$ potential hosts brighter than $M_{R}=-20$, resulting in an AGN fraction of $f_{A}\left(L_{\mathrm{X}}>\right.$ $\left.10^{43} ; M_{R}<-20\right)=0.020_{-0.008}^{+0.012}$. To calculate the $R_{200}$ radii of the three clusters in the $\mathrm{Cl1604}$ system which have associated AGNs, we make use of the velocity dispersions, $\sigma_{v}$, measured by G08 and the equation $R_{200}=2 \sigma_{v} /[\sqrt{200} H(z)]$. The resulting $R_{200}$ radii range from 0.52 to $1.01 h_{70}^{-1} \mathrm{Mpc}$ (or 1 . 11 to 2 !.18 on the sky). Within these radii we find three supercluster AGNs, although only two have $2-10 \mathrm{keV}$ X-ray luminosities greater than $10^{43} h_{70}^{-2} \operatorname{erg~s}^{-1}$. These are sources 7 and 8 in Clusters B and $\mathrm{D}$.

To determine which nonactive cluster galaxies are capable of hosting a detectable AGN we construct a flux limit map covering our entire ACIS-I mosaic. This map takes into account the local background level, the size of the PSF and the detector vignetting at the location of each cluster member to determine the faintest detectable AGN at that position; details on its construction are given in K09. Within the $R_{200}$ radii of Clusters B and D we find 74 galaxies brighter than $M_{R}=-20$ within which both AGNs could have been detected. Rest-frame magnitudes for the cluster members were calculated from our observed magnitudes in the F814W band using an Sb galaxy $k$-correction. ${ }^{11}$

The resulting AGN fraction amounts to two AGNs in 74 possible hosts or $f_{A}\left(L_{\mathrm{X}}>10^{43} ; M_{R}<-20\right)=0.027_{-0.020}^{+0.045}$ (the uncertainties correspond to $90 \%$, one-sided Poisson confidence limits). An important point to note is that we have treated the diffuse emission from Cluster B as an enhanced local background

\footnotetext{
11 We also convert our magnitudes from the $\mathrm{AB}$ to the Vega system using the appropriate ACS zeropoint in order to match the magnitude limit of Eastman et al. (2007).
}

in constructing our flux limit map. Although this emission effectively raises our point source detection threshold in the soft band, the emission does not affect our ability to detect sources in the hard band. We calculate that both AGNs detected within the $R_{200}$ radius of Clusters $\mathrm{B}$ and $\mathrm{D}$ would have been detected in all of the cluster members with $M_{R}<-20$ out to the same radius. If we instead wish to be conservative and remove all galaxies embedded in the cluster's diffuse emission, which extends roughly $50^{\prime \prime}$, this reduces the number of potential hosts by 14 , resulting in an AGN fraction of $f_{A}\left(L_{\mathrm{X}}>10^{43} ; M_{R}<-20\right.$; no Cluster B) $=0.033_{-0.025}^{+0.055}$.

Our measured cluster AGN fraction within $R_{200}$ at $z \sim 0.9$ is significantly higher than the fraction measured at low-redshift by Martini et al. (2006), who found $f_{A}\left(L_{\mathrm{X}}>10^{43} ; M_{R}<-20\right)=$ $0.0007_{-0.0007}^{+0.0021}$, but largely consistent with the moderate-redshift results of Eastman et al. (2007). Even if we exclude galaxies embedded within the diffuse emission of Cluster B, we do not find a statistically significant increase over the fraction measured at $z \sim 0.6$ given our large errors $\left(0.033_{-0.025}^{+0.055}\right.$ versus $\left.0.020_{-0.008}^{+0.012}\right)$. Therefore we cannot report a substantial change in AGN activity within the $R_{200}$ radius from $z \sim 0.6$ to $z \sim 0.9$. This is perhaps not surprising since, as we discussed in Section 4.4, most of the AGNs detected in the supercluster (6 of 9) are found in intermediate density environments and well outside the $R_{200}$ radius of its constituent clusters. A much larger sample of AGNs in $z \sim 1$ clusters will be needed in order to determine whether the AGN fraction increases substantially beyond the levels observed at moderate redshifts.

\section{DISCUSSION}

In many cases, the observed properties of the nine AGN hosts found in the $\mathrm{Cl1604}$ supercluster either confirm the findings of previous wide-field galaxy surveys or extend to higher redshifts trends observed in lower-redshift structures. With regard to overall host morphology, optical luminosity and color, our findings are generally consistent with the results of several past studies which find host galaxies to be largely massive, early-type galaxies showing signs of recent star formation (Kauffmann et al. 2003; Heckman 2004; Sánchez et al. 2004; Nandra et al. 2007; Silverman et al. 2008). The fact that our supercluster AGNs are found in luminous spheroids which have bluer colors than similar nonactive galaxies is not surprising as it is precisely these galaxies that contain the two ingredients required for nuclear activity: a massive black hole and an abundant gas supply (Kauffmann et al. 2003). Our finding of predominantly blue-cored, early-type hosts agrees well with the growing consensus that powerful AGNs are located in massive galaxies with bulges containing younger stellar populations.

Our results also seem to confirm the increased post-starburstAGN connection reported at higher redshifts. While there is extensive evidence for younger stellar ages and recent starburst activity in moderate-luminosity AGNs (Schmitt et al. 1999; González Delgado et al. 2001; Cid Fernandes et al. 2001; Kauffmann et al. 2003; Heckman 2004; Ho 2005), the fraction of Seyferts associated with post-starburst hosts at low redshift remains fairly small. Goto (2006) found that although poststarburst hosts were more often associated with AGNs than normal galaxies, hosts exhibiting strong post-starburst spectral signatures were relatively rare, accounting for only $4.2 \%$ of narrow-line type 2 Seyferts in the SDSS sample. Recently, Georgakakis et al. (2008) reported an increase in this fraction at higher redshifts $(0.68 \leqslant z \leqslant 0.88)$ using a sample of $\mathrm{X}$ ray sources detected in the Extended Groth Strip. They find 
that $21 \%$ of AGN hosts located on the red sequence exhibit post-starburst signatures. In the $\mathrm{Cl} 1604$ supercluster, we find a significant fraction $(\sim 44 \%)$ of all host spectra show poststarburst features, supporting the notion of an increased poststarburst-AGN association at redshifts approaching unity. Of course, we must use caution when directly comparing these results as we have assumed that at least some AGN emission contributes to our observed [O II] lines whereas previous studies have not. In fact Yan et al. (2006) warn that AGN-driven [O II] emission being mistaken for ongoing star formation may have led previous studies to underestimate the prevalence of the poststarburst-AGN connection at lower redshifts.

On one important topic, merger activity as a triggering mechanism, our observations do seem to be at odds with previous studies. Although luminous QSO activity has been tied to merger activity (Canalizo \& Stockton 2001; Bennert et al. 2008), a similar link has not been clearly established with moderate-luminosity Seyferts (De Robertis et al. 1998; Grogin et al. 2003, 2005; Pierce et al. 2007). In particular Grogin et al. (2005) found that while higher-redshift hosts are bulge dominated, their asymmetry and near-neighbor counts were consistent with non-AGN control samples. We find that although only two of eight hosts have asymmetry and concentration parameters indicative of ongoing mergers, several galaxies do show signs of recent interactions (i.e., tidal features or assymetric/multiple cores). Many of these hosts appear otherwise relaxed (being fit with high Sersic indices, for example), implying that any merger activity was either fairly minor so as to not disrupt the galaxy significantly or enough time has transpired for the system to have sufficiently relaxed. That being said, we note once again that the asymmetry of the single AGN that we find in an apparent merger remnant was not greatly enhanced by the presence of faint tidal features largely due to its considerably brighter and compact nucleus.

Overall we find that the properties of the nine host galaxies are generally consistent with a scenario in which recent interactions have triggered both increased levels of nuclear activity and an enhancement of centrally concentrated star formation, followed by a rapid truncation of the latter. This is evidenced by the post-starburst signatures in several host spectra, their blue-cored color morphologies, and the high fraction of hosts that show signs of recent interactions. Whether the star formation and nuclear activity were triggered simultaneously or with some delay remains to be determined, but the AGN emission does seem to persists beyond the termination of starburst activity in these systems. Given this fact, our observations are therefore consistent with models in which feedback from the AGN itself is responsible for this suppression and the subsequent migration of these galaxies from the blue cloud to the red sequence. We do find that a majority of the host galaxies have intermediate colors that place them in the transition zone of the CMD, which further supports this scenario. This is in agreement with several recent studies which have also found that the color of host galaxies cover a similar range, extending from the red edge of the blue cloud to the red sequence (Sánchez et al. 2004; Nandra et al. 2007; Martin et al. 2007; Georgakakis et al. 2008; Silverman et al. 2008; although see Westoby et al. 2007 for a differing conclusion).

Of particular interest for our study are the results of Silverman et al. (2008), who found that within a redshift interval containing two large-scale structures in the E-DF-S $(0.63 \leqslant z \leqslant 0.76)$ AGN hosts are preferentially located in the transition zone. We find a similar result within the $\mathrm{Cl} 1604$ complex, where a majority of host galaxies are located between the blue cloud and the red sequence. These results suggest that the hosts of moderateluminosity AGNs are predominantly a transition population within these structures, caught in the process of migrating from blue to red. Coupled with recent findings that AGNs are more common in richer environments than they are in the field (Gilli et al. 2003; Cappelluti et al. 2005; Eastman et al. 2007; K09), this finding implies that AGN-related feedback may play an important role in accelerating galaxy evolution in large-scale structures.

That being said, we should note that in studying the lowredshift supercluster A901/902, Gilmour et al. (2007) found many AGN hosts with colors similar to that of the supercluster red sequence. Likewise Nandra et al. (2007) and Silverman et al. (2008) found a significant population of field AGNs associated with redder hosts. As Silverman et al. point out, most of these are found at lower-redshifts where the strong color evolution of massive galaxies capable of hosting AGNs insures these galaxies will be redder than those found at $z=0.9$. We would like to further point out that most of these galaxies harbor AGNs less luminous than those detected in the Cl1604 supercluster. For example, a source detected at our $3 \sigma$ flux limit would have a rest-frame $0.5-8 \mathrm{keV}$ luminosity of $\sim 8 \times 10^{42} h_{70}^{-2} \mathrm{erg} \mathrm{s}^{-1}$ at the average redshift of the $\mathrm{Cl} 1604$ supercluster. All of the X-ray sources detected by Gilmour et al. (2007) have luminosities below this limit. Likewise most of the sources detected by Nandra et al. (2007) on the red sequence have luminosities below $10^{43} h_{70}^{-2} \mathrm{erg} \mathrm{s}^{-1}$; only one of our AGN fall into this regime. This is not extremely surprising as it has been observed for some time that less luminous AGNs have older stellar populations than their more powerful counterparts (e.g., Kauffmann et al. 2003; Heckman 2004). The fact that we do not observe any moderateluminosity AGN on the red sequence is consistent with the AGN feedback model of Hopkins et al. (2005) which suggests that AGNs should become less luminous as their hosts migrate from the blue cloud to the red sequence due to the depletion of material which would otherwise feed the central black hole.

An alternative theory that is also consistent with our observations is one of episodic star formation and AGN activity in red sequence galaxies. In this scenario, minor mergers that do not significantly disrupt the galaxy initiate nuclear activity and trigger renewed star formation which temporarily moves the system off the supercluster red sequence. This produces a population of young, blue stars we observe as the blue cores of these systems in a process similar to the one described by Menanteau et al. (2001b). This results in a build-up of both stellar bulge mass and black hole mass that is eventually regulated by the feedback mechanism. As star formation is quenched, the newly formed stars fade and the galaxy returns to the red sequence but this time with a higher luminosity. In this view, AGN hosts in the supercluster are not in fact transitioning from blue to red, but instead from low to high luminosity (mass) on the red sequence. This scenario does seem to be supported by the relatively red colors we find in many of the blue-cored host galaxies. If it were not for their cores, these galaxies would be on the red sequence. Of course in order for this process to work, either the red sequence galaxies would need to retain a significant reservoir of cold gas to fuel future activity or a sufficient amount would need to be accreted from the merging, presumable gas-rich, galaxy. Possible methods to distinguish this scenario from that of a major-merger driven framework include searching for low-surface brightness merger signatures with deeper high-resolution imaging (e.g., Bennert et al. 2008) and detailed stellar population modeling using higher signal-to-noise spectra, which may reveal the 
presence of a young stellar component on top of a predominately old population (e.g., Serra \& Trager 2007). Both of these would be difficult at best to employ given the redshift of the Cl1604 system.

\section{CONCLUSIONS}

In summary, we find that the $\mathrm{X}$-ray-selected AGNs detected in the Cl1604 supercluster are largely hosted by luminous $\left(M_{V}<\right.$ -21.1 ), spheroidal, and/or bulge dominated galaxies which are bluer than similar galaxies on the system's red sequence. The integrated colors of a majority of the hosts $(5 / 8)$ place them in the valley between the supercluster red sequence and the more diffuse blue cloud. In half of the hosts, the bluer overall colors are the result of blue resolved cores in otherwise red spheroids. Given that the supercluster AGN luminosities do not reach the QSO level and the lack of obvious AGN contamination in their optical spectra, we interpret the blue cores as the result of recent star formation. Many of these galaxies do show signs of starburst activity within the last $\sim 1 \mathrm{Gyr}$ as $\sim 44 \%$ (4/9) of the host spectra exhibit either strong $\mathrm{H}-\delta$ absorption or a pronounced Balmer series. We also find a majority of hosts (6/9) show signs of recent or pending interactions, a possible indication of their triggering mechanism. With regard to environment, we observe that the hosts tend to avoid the densest regions of the supercluster and are instead located in intermediate density environments, such as the infall region of a massive cluster or in poorer systems undergoing assembly. Finally, we measure an increased fraction of supercluster members that harbor AGN relative to low-redshift $(z<0.3)$ clusters, but we do not observe a substantial change from the fraction reported at $z \sim 0.6$.

In general, our observations are fairly consistent with theories that link AGN activity to galaxies undergoing transformation from star-forming systems in the blue cloud to passively evolving galaxies on the red sequence. This is evidenced by the fact that (1) a significant fraction of the AGN hosts are located in the transition zone of the CMD, which is normally sparsely populated, (2) several hosts show evidence of a recent enhancement of star formation that was abruptly terminated, and (3) there are indications of recent merger activity in many of the hosts, which are located in moderate-density environments thought to be conducive to galaxy interactions. An alternative theory that is also consistent with our observations is one of episodic star formation and AGN activity in red sequence galaxies triggered by galaxy interactions. In this scenario, AGN hosts in the supercluster are not in fact transitioning from blue to red, but instead from low to high luminosity (mass) on the red sequence.

Many of these conclusions, especially those regarding the post-starburst-AGN connection, depend on our assumption that the [O II] emission lines seen in the host galaxies are due in part to AGN emission. We plan to test this assumption in the near future with near-infrared spectroscopic observations of each AGN host in order to obtain $\mathrm{H} \alpha$ and [N II] EWs and line ratios. The observations of one host which have already been completed have confirmed that AGN emission contributes to the galaxies [O II] line flux, leading to an overestimated SFR. If we find similar results for the remaining hosts, this will help confirm that a significant fraction of AGNs in the Cl1604 supercluster have experienced a recent and rapid truncation of their star formation activity.

This work is supported by the Chandra General Observing Program under award number GO6-7114X. Additional support for this program was provided by NASA through a grant HSTGO-11003 from the Space Telescope Science Institute, which is operated by the Association of Universities for Research in Astronomy, Inc. The spectrographic data used herein were obtained at the W. M. Keck Observatory, which is operated as a scientific partnership among the California Institute of Technology, the University of California and the National Aeronautics and Space Administration. The Observatory was made possible by the generous financial support of the W. M. Keck Foundation.

\section{REFERENCES}

Abraham, R. G., van den Bergh, S., \& Nair, P. 2003, ApJ, 588, 218

Baldry, I. K., Glazebrook, K., Brinkmann, J., Ivezić, Z., Lupton, R. H., Nichol, R. C., \& Szalay, A. S. 2004, ApJ, 600, 681

Barger, A. J., Cowie, L. L., Mushotzky, R. F., Yang, Y., Wang, W.-H., Steffen, A. T., \& Capak, P. 2005, AJ, 129, 578

Barger, A. J., et al. 2003, AJ, 126, 632

Barnes, J. E., \& Hernquist, L. E. 1991, ApJ, 370, L65

Bauer, F. E., Alexander, D. M., Brandt, W. N., Hornschemeier, A. E., Vignali, C., Garmire, G. P., \& Schneider, D. P. 2002, AJ, 124, 2351

Bennert, N., Canalizo, G., Jungwiert, B., Stockton, A., Schweizer, F., Peng, C. Y., \& Lacy, M. 2008, ApJ, 677, 846

Bertin, E., \& Arnouts, S. 1996, A\&AS, 117, 393

Boyle, B. J., \& Terlevich, R. J. 1998, MNRAS, 293, L49

Branchesi, M., Gioia, I. M., Fanti, C., Fanti, R., \& Cappelluti, N. 2007, A\&A, 462, 449

Brusa, M., et al. 2005, A\&A, 432, 69

Butcher, H., \& Oemler, A., Jr. 1984, ApJ, 285, 426

Canalizo, G., \& Stockton, A. 2001, ApJ, 555, 719

Cappelluti, N., Cappi, M., Dadina, M., Malaguti, G., Branchesi, M., D’Elia, V., \& Palumbo, G. G. C. 2005, A\&A, 430, 39

Cappi, M., et al. 2001, ApJ, 548, 624

Cavaliere, A., Colafrancesco, S., \& Menci, N. 1992, ApJ, 392, 41

Cid Fernandes, R., Heckman, T., Schmitt, H., Delgado, R. M. G., \& StorchiBergmann, T. 2001, ApJ, 558, 81

Ciliegi, P., Vignali, C., Comastri, A., Fiore, F., La Franca, F., \& Perola, G. C. 2003, MNRAS, 342, 575

De Robertis, M. M., Yee, H. K. C., \& Hayhoe, K. 1998, ApJ, 496, 93

de Vaucouleurs, G. 1948, Ann. d'Astrophys., 11, 247

Dickey, J. M., \& Lockman, F. J. 1990, ARA\&A, 28, 215

Dressler, A. 1980, ApJ, 236, 351

Driver, S. P., et al. 2006, MNRAS, 368, 414

Eastman, J., Martini, P., Sivakoff, G., Kelson, D. D., Mulchaey, J. S., \& Tran, K.-V. 2007, ApJ, 664, L9

Faber, S. M., et al. 2003, in Proc. SPIE 4841, Instrument Design and Performance for Optical/Infrared Ground-based Telescopes, ed. M. Iye \& A. F. M Moorwood (Bellingham, WA: SPIE), 1657

Faber, S. M., et al. 2007, ApJ, 665, 265

Fisher, D., Fabricant, D., Franx, M., \& van Dokkum, P. 1998, ApJ, 498, 195

Gal, R. R., Lemaux, B. C., Lubin, L. M., Kocevksi, D., \& Squires, G. K. 2008, ApJ, 684, 933

Gal, R. R., \& Lubin, L. M. 2004, ApJ, 607, L1

Gal, R. R., Lubin, L. M., \& Squires, G. K. 2005, AJ, 129, 1827

Garmire, G. P., Bautz, M. W., Ford, P. G., Nousek, J. A., \& Ricker, G. R., Jr. 2003, in Proc. SPIE 4851, X-Ray and Gamma-Ray Telescopes and Instruments for Astronomy, ed. J. E. Truemper \& H. D. Tananbaum (Bellingham, WA: SPIE), 28

Gebhardt, K., et al. 2000, ApJ, 539, L13

Georgakakis, A., et al. 2007, ApJ, 660, L15

Georgakakis, A., et al. 2008, MNRAS, 385, 2049

Gerke, B. F., et al. 2007, ApJ, 660, L23

Gilli, R., et al. 2003, ApJ, 592, 721

Gilmour, R., Gray, M. E., Almaini, O., Best, P., Wolf, C., Meisenheimer, K., Papovich, C., \& Bell, E. 2007, MNRAS, 380, 1467

Gómez, P. L., et al. 2003, ApJ, 584, 210

González Delgado, R. M., Heckman, T., \& Leitherer, C. 2001, ApJ, 546, 845

Goto, T. 2006, MNRAS, 369, 1765

Grogin, N. A., et al. 2003, ApJ, 595, 685

Grogin, N. A., et al. 2005, ApJ, 627, L97

Gu, Q. S., Huang, J. H., de Diego, J. A., Dultzin-Hacyan, D., Lei, S. J., \& Benítez, E. 2001, A\&A, 374, 932 
Heckman, T. M. 2004, in Coevolution of Black Holes and Galaxies, ed. L. C. Ho (Cambridge: Cambridge Univ. Press), 358

Henry, J. P., \& Briel, U. G. 1991, A\&A, 246, L14

Ho, L. C. 2005, arXiv:astro-ph/0511157

Hopkins, P. F., Bundy, K., Hernquist, L., \& Ellis, R. S. 2007, ApJ, 659, 976

Hopkins, P. F., Hernquist, L., Martini, P., Cox, T. J., Robertson, B., Di Matteo, T., \& Springel, V. 2005, ApJ, 625, L71

Kartaltepe, J. S., et al. 2007, ApJS, 172, 320

Kauffmann, G., et al. 2003, MNRAS, 346, 1055

Kennicutt, R. C., Jr. 1998, ARA\&A, 36, 189

Kocevski, D. D., Lubin, L. M., Gal, R., Lemaux, B. C., Fassnacht, C. D., \& Squires, G. K. 2009, ApJ, 690, 295

Koekemoer, A. M., Fruchter, A. S., Hook, R. N., \& Hack, W. 2002, in The 2002 HST Calibration Workshop: Hubble After the Installation of the ACS and the NICMOS Cooling System, ed. S. Arribas, A. Koekemoer, \& B. Whitmore (Baltimore, MD: STScI), 337

Lewis, I., et al. 2002, MNRAS, 334, 673

Lin, R. 2007, Master's thesis, Univ. of California, Davis

Lisker, T. 2008, ApJS, 179, 319

Lotz, J. M., Primack, J., \& Madau, P. 2004, AJ, 128, 163

Lubin, L. M., Postman, M., Oke, J. B., Ratnatunga, K. U., Gunn, J. E., Hoessel, J. G., \& Schneider, D. P. 1998, AJ, 116, 584

Mainieri, V., et al. 2008, ApJS, 179, 95

Marshall, P. J., et al. 2009, ApJ, 694, 924

Martin, D. C., et al. 2007, ApJS, 173, 342

Martini, P., Kelson, D. D., Kim, E., Mulchaey, J. S., \& Athey, A. A. 2006, ApJ, 644, 116

Menanteau, F., Abraham, R. G., \& Ellis, R. S. 2001a, MNRAS, 322, 1

Menanteau, F., Jimenez, R., \& Matteucci, F. 2001b, ApJ, 562, L23

Mihos, J. C., \& Hernquist, L. 1996, ApJ, 464, 641

Monet, D. G., et al. 2002, VizieR Online Data Catalog, 1284, 0

Nandra, K., et al. 2007, ApJ, 660, L11
Oke, J. B., Postman, M., \& Lubin, L. M. 1998, AJ, 116, 549

Oke, J. B., et al. 1995, PASP, 107, 375

Peng, C. Y., Ho, L. C., Impey, C. D., \& Rix, H.-W. 2002, AJ, 124, 266

Pierce, C. M., et al. 2007, ApJ, 660, L19

Poggianti, B. M., \& Barbaro, G. 1997, A\&A, 325, 1025

Poggianti, B. M., Smail, I., Dressler, A., Couch, W. J., Barger, A. J., Butcher, H., Ellis, R. S., \& Oemler, A. J. 1999, ApJ, 518, 576

Postman, M., Lubin, L. M., \& Oke, J. B. 1998, AJ, 116, 560

Postman, M., et al. 2005, ApJ, 623, 721

Ruderman, J. T., \& Ebeling, H. 2005, ApJ, 623, L81

Sánchez, S. F., et al. 2004, ApJ, 614, 586

Schmitt, H. R., Storchi-Bergmann, T., \& Fernandes, R. C. 1999, MNRAS, 303, 173

Serra, P., \& Trager, S. C. 2007, MNRAS, 374, 769

Sersic, J. L. 1968, Atlas de Galaxias Australes (Cordoba, Argentina: Observatorio Astronomico)

Silverman, J. D., et al. 2005, ApJ, 618, 123

Silverman, J. D., et al. 2008, ApJ, 675, 1025

Smail, I., Dressler, A., Couch, W. J., Ellis, R. S., Oemler, A. J., Butcher, H., \& Sharples, R. M. 1997, ApJS, 110, 213

Smith, G. P., Treu, T., Ellis, R. S., Moran, S. M., \& Dressler, A. 2005, ApJ, 620 78

Springel, V., Di Matteo, T., \& Hernquist, L. 2005, MNRAS, 361, 776

Somerville, R. S., Hopkins, P. F., Cox, T. J., Brant, E. R., \& Hernquist, L. 2008, MNRAS, 391, 481

Sutherland, W., \& Saunders, W. 1992, MNRAS, 259, 413

Taylor, E. L., et al. 2005, MNRAS, 361, 1352

Tremaine, S., et al. 2002, ApJ, 574, 740

Treu, T., et al. 2005, ApJ, 633, 174

Westoby, P. B., Mundell, C. G., \& Baldry, I. K. 2007, MNRAS, 382, 1541

Yan, R., Newman, J. A., Faber, S. M., Konidaris, N., Koo, D., \& Davis, M. 2006, ApJ, 648, 281 